\title{
Expression profile analysis of microRNAs and downregulated miR-486-5p and miR-30a-5p in non-small cell lung cancer
}

\author{
JIANJIE ZHU ${ }^{1,2^{*}}$, YUANYUAN ZENG ${ }^{1,2^{*}}$, CHUN XU $^{3 *}$, HUALONG QIN ${ }^{3}$, \\ ZHE LEI ${ }^{4}$, DAN SHEN ${ }^{1}$, ZEYI LIU ${ }^{1,2,4}$ and JIAN-AN HUANG ${ }^{1,2}$ \\ ${ }^{1}$ Department of Respiratory Medicine, The First Affiliated Hospital of Soochow University; ${ }^{2}$ Institute of Respiratory Diseases, \\ Soochow University; ${ }^{3}$ Department of Cardiothoracic Surgery, The First Affiliated Hospital of Soochow University; \\ ${ }^{4}$ Suzhou Key Laboratory for Molecular Cancer Genetics, Suzhou, Jiangsu, P.R. China
}

Received May 27, 2015; Accepted June 17, 2015

DOI: $10.3892 /$ or.2015.4141

\begin{abstract}
Lung cancer is the leading cause of cancer-related mortality worldwide and although there have been improvements in treatment there is a low survival rate. The aim of the present study was to investigate the effect of microRNA (miRNA) on cell pathways. A miRNA microarray was used to profile miRNAs of lung cancer tissues. It was identified that 33 miRNAs with $>2.0$-fold change and FDR $<0.05$ were differentially expressed between the adjacent noncancerous lung tissues and non-small cell lung cancers NSCLCs $(\mathrm{P}<0.005)$. The data were optimized in combination with physical interaction analysis to obtain crucial miRNAs. The results showed that differentially expressed miRNAs were associated with biological processes such as cell migration, protein phosphorylation and neuron differentiation, and signaling pathways such as MAPK, TGF- $\beta$ and PI3K/ Akt signaling pathways. Validation of significant miRNAs in independent 40 paired NSCLC tissues demonstrated that the expression level of miR-486-5p and miR-30a-5p was significantly downregulated in another 40 paired lung cancer tissues. Taken together, the results provided strong evidence of the possible involvement of miRNAs in the development and progression of NSCLC. Thus, the results are of importance for clinical investigators and for those who design miRNA-based novel cancer therapeutics.
\end{abstract}

Correspondence to: Professor Jian-An Huang or Dr Zeyi Liu, Department of Respiratory Medicine, The First Affiliated Hospital of Soochow University, 188 Shizi Street, Suzhou, Jiangsu 215123, P.R. China

E-mail: huang_jian_an@yeah.net

E-mail: liuzeyisuda@163.com

${ }^{*}$ Contributed equally

Key words: microRNAs, signaling pathway, non-small cell lung cancer

\section{Introduction}

Lung cancer is the leading cause of cancer-related mortality worldwide (1), with non-small cell lung cancer (NSCLC) accounting for $80 \%$ of all cases of lung cancer. Despite improvement in cancer treatment, the 5-year survival rate remains at $<10 \%$. However, diagnosis followed by treatment in the form of surgery at early stage may lead to a 5-year survival rate of $55-80 \%$ (2). Therefore, preferential understanding of the mechanisms of tumor development and progression are of great importance in early detection and prevention as well as the targeted treatment of NSCLC.

MicroRNA (miRNA) are a class of small non-coding RNAs between 19 and 24 nt that can repress translation or promote the degradation of target mRNAs $(3,4)$. It has been confirmed that approximately $30 \%$ of human protein-coding genes are regulated by miRNAs (5). Thus, miRNAs play an important role in regulating various biological processes, such as cell proliferation (6), differentiation (7) and apoptosis (8). Previous findings showed that an altered expression of miRNAs in cancer relative to corresponding normal tissues, and specific expression signatures or panels can classify human cancers (9), distinguish tumor subtype (10), and are closely associated with prognosis (11). In a recent study, it was shown that altered miRNAs can regulate epithelial-to-mesenchymal transition (EMT) in tumor progression (12). Moreover, increasing evidence suggested that dysregulation of miRNAs contributed to stem cell fate (13).

hsa-miR-486-5p is located on chromosome 8p11.21, which is one of the most frequent genomic deletion regions that contain potential tumor-suppressor genes in various types of tumors, such as NSCLC $(14,15)$. hsa-miR-486-5p was first cloned from the fetal liver. Additionally, a reduced miR-486-5p expression is a frequent molecular event in human malignances (16-19).

hsa-miR-30a-5p is a member of the microRNA-30 family, and is located on chromosome $6 \mathrm{q} 13$, which has been reported to be a genomic fragile region due to loss of heterozygosity in breast and lung cancer $(20,21)$. miR-30a-5p has received more attention due to its important role in various biological and pathological processes, including development, differentiation, autophage and apoptosis $(22,23)$. 
In the present study, the microRNA microarray was employted to profile miRNA of lung cancer tissues from Chinese subjects. The data were optimized in combination with physical interaction analysis to obtain crucial miRNAs. The results showed the expression level of miR-486-5p and miR-30a-5p was significantly downregulated in another 40 paired lung cancer tissues.

\section{Materials and methods}

Patients and tissue samples. Forty-four paired NSCLC and adjacent non-cancerous lung tissues were collected at the time of surgery and before chemotherapy. The samples were obtained from patients at the First Affiliated Hospital of the Soochow University between 2007 and 2013. Informed consent was provided the patients participating in the study. Histological and pathological diagnostics of tissue samples from the patients were obtained from the Department of Pathology according to the Revised International System for Staging Lung Cancer. Tissue samples were snap-frozen and stored in an cryofreezer at $-80^{\circ} \mathrm{C}$. Study approval was obtained by the Ethics Committee of the First Affiliated Hospital of Soochow University.

Analysis of miRNA microarray. miRNA microarray assays were performed using the Agilent Human miRNA microarray platform (Agilent Technologies, Santa Clara, CA, USA) at Shanghai Biochip Co., Ltd. (Shanghai, China). The microarray containing probes for 2006 human miRNAs from the Sanger database V19.0. Total RNA (100 ng) derived from cells was labeled with $\mathrm{Cy} 3$. Microarray slides were scanned using an Agilent microarray scanner. Labeling and hybridization were performed according to the instructions in the Agilent microRNAs microarray system. The microarray image information was converted into spot intensity values using Feature Extraction software 10.7 (Agilent Technologies). The signal after background subtraction was exported directly into the GeneSpring software 11.0 (Agilent Technologies) for quantile normalization. Log transformation with base 2 was then performed. The limma algorithm (24) was then applied to filter the differentially expressed genes, after the significant analysis and FDR analysis (25) under the specified criteria: i), fold change $>2$ or $<0.5$; and ii), FDR $<0.05$. Targetscan 6.1 was utilized as the tool for predicting miRNA target on the differentially expressed miRNA.

Gene ontology (GO) analysis. GO analysis was performed to facilitate elucidating the biological implications of unique genes in the significant or representative profiles of the target gene of the differentially expressed miRNA in the experiment (26). The GO annotations were downloaded from NCBI (http://www.ncbi.nlm.nih.gov/), UniProt (http://www.uniprot. org/) and the Gene Ontology (http://www.geneontology.org/). Fisher's exact test was applied to identify the significant GO categories and FDR was used to correct the p-values.

GO-Tree. The Gene Ontology is structured as a directed acyclic graph, and each term has defined relationships to one or more other terms. GO-Tree is constructed based on the Gene Ontology Directed Acyclic Graph to provide user friendly data navigation and visualization. The significant GO-Term
$(\mathrm{P}<0.01)$ in $\mathrm{GO}$ analysis was selected based on the up and down differentially expressed genes to construct the GO-Tree to summarize the function affected in the experiment (27).

Pathway analysis. Pathway analysis was used to identify the significant pathway of the differential genes according to the KEGG database. The Fisher's exact test was used to select the significant pathway, and the threshold of significance was defined by $\mathrm{p}$-value and FDR (28).

Path-act-network. KEGG included metabolism, membrane transport, signal transduction and cell cycle pathways. Genes in the enriched biological pathway were selected $(\mathrm{P}<0.05)$ and Cytoscape (29) was used for the graphical representations of pathways.

RNA isolation, cDNA synthesis, and reverse transcriptasequantitative polymerase chain reaction $(q R T-P C R)$. Total RNA was extracted from tissues using an HP Total RNA kit (Omega Biotech, Stamford, CT, USA) according to the manufacturer's instructions. The amount of RNA was measured on a NanoDrop (Thermo Fisher Scientific, Waltham, MA, USA). Synthesis of cDNA with reverse transcriptase (RT) was performed with a M-MLV First Strand kit (Life Technologies, Gaithersburg, MD, USA). Primer sequences for miR-486-5p, miR-30a-5p and U6 detection were obtained from Riobobio (Guangzhou, China). The RT primer for mature miRNAs and U6 was designed according to the concept of a stem-loop RT primer (30). RT-qPCR analysis was carried out using Platinum SYBR-Green qPCR SuperMix-UDG kits (Life Technologies) according to the manufacturer's instructions. Real-time PCR was performed on an ABI Prism 7500 Real-Time PCR system (Applied Biosystems, Foster City, CA, USA). Ct values of miRNAs were equilibrated to U6, which were used as internal controls. Relative expression was calculated using the $\Delta \Delta \mathrm{C}_{\mathrm{t}}$ method.

Statistical analysis. Differences in miR-486-5p and miR-30a-5p expression between NSCLC (T) and adjacent paired noncancerous lung tissues $(\mathrm{N})$ were analyzed using a paired t-test (two-tailed) by GraphPad Prism 5.0 (GraphPad, San Diego, CA, USA). The Fisher's exact was applied to identify the significant GO categories and FDR was used to correct the p-values. The Chi-square test was used with SPSS16.0 software to test independence between two variables (SPSS, Chicago, IL, USA).

\section{Results}

Differentially expressed miRNAs in NSCLC. We applied the limma algorithm to filter the differentially expressed genes, followed by the significant analysis and FDR analysis under the specified criteria: i), fold change $>2$ or $<0.5$; and ii), FDR $<0.05$. The data from miRNA expression profiling indicated that 33 miRNAs with $>2.0$-fold change and FDR $<0.05$ were differentially expressed between the lung normal tissue and NSCLCs $(\mathrm{P}<0.005)$, with a higher expression in NSCLC for 15 miRNAs and a lower expression for 18 miRNAs (Fig. 1). Among the miRNAs with a decreased expression, miR-486-5p and miR-30a-5p were identified as the most significantly downregulated, respectively, by bioinformatics. 


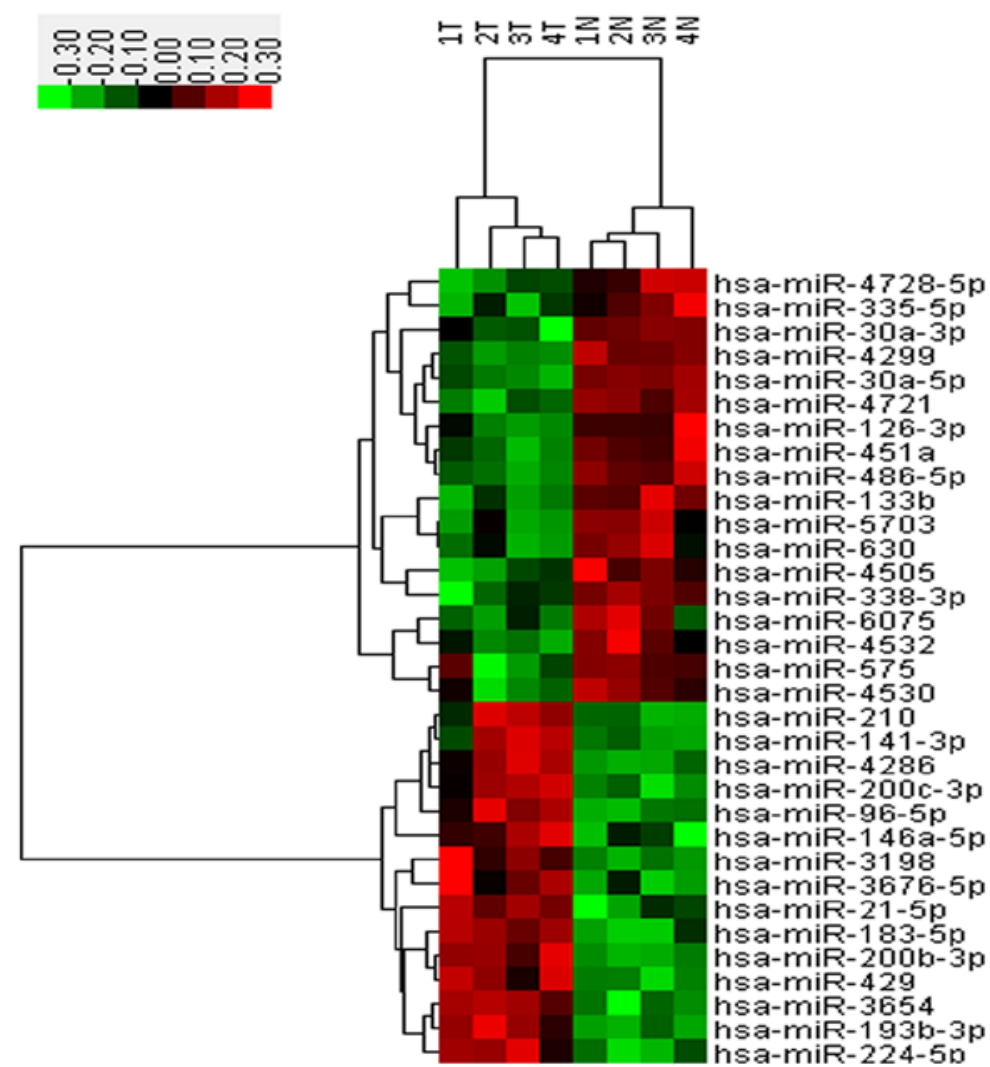

Figure 1. The miRNAs differentially expressed in lung cell carcinoma vs. paired normal tissues. Hierarchical clustering of 33 miRNA genes with a significantly different expression, i.e., i) fold change $>2$ or $<0.5$ and ii) FDR $<0.05$ and $\mathrm{P}<0.005$. Rows are individual microRNA; columns are tumor and normal tissue samples. The color scale is the relative expression ratio of a miRNA following normalization (red, high expression level; green, low expression level).

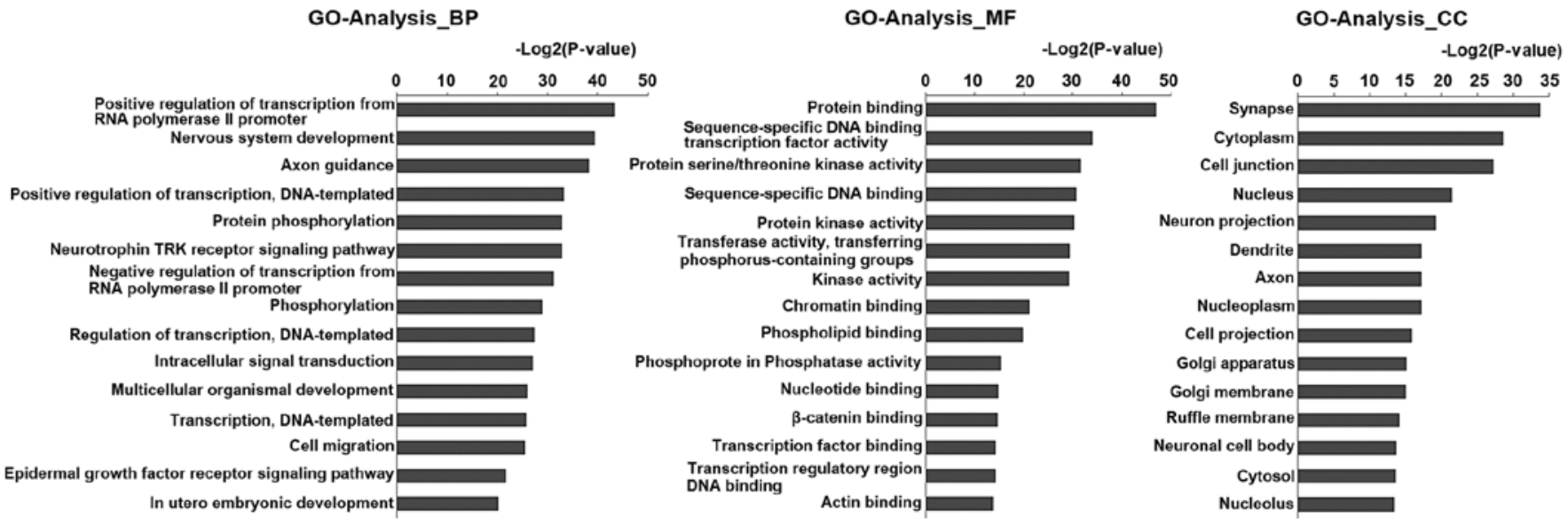

Figure 2. Fisher's exact test was applied to identify the significant GO categories and FDR was used to correct the p-value. GO, Gene Ontology; BP, biological process ; MF, molecular function; CC, cellular component.

Physical interaction analysis. Based on the miRNA array data, to identify the effect of the differentially expressed miRNA towards the biological process and signal transduction on the lung cancer, GO analysis was performed to facilitate elucidating the biological implications of unique genes in the significant or representative profiles of the target gene of the the differentially expressed miRNA in the experiment (Fig. 2), The Gene Ontology is structured as a directed acyclic graph, and each term has defined relationships to one or more other terms. The GO-Tree is constructed based on the Gene Ontology
Directed Acyclic Graph to provide user friendly data navigation and visualization. The significant GO-Term $(\mathrm{P}<0.01)$ in GO analysis was selected based on the up and down differentially expressed genes to construct the GO-Tree to summarize the function affected in the experiment (Fig. 3).

It has been widely accepted that in solid tumors miRNAs are deregulated, suggesting their involvement in crucial cell pathways including cell-cell adhesion and signaling, cell cycle regulation and apoptosis, which play a significant role in the pathogenesis of cancer. Enriched KEGG and pathway 


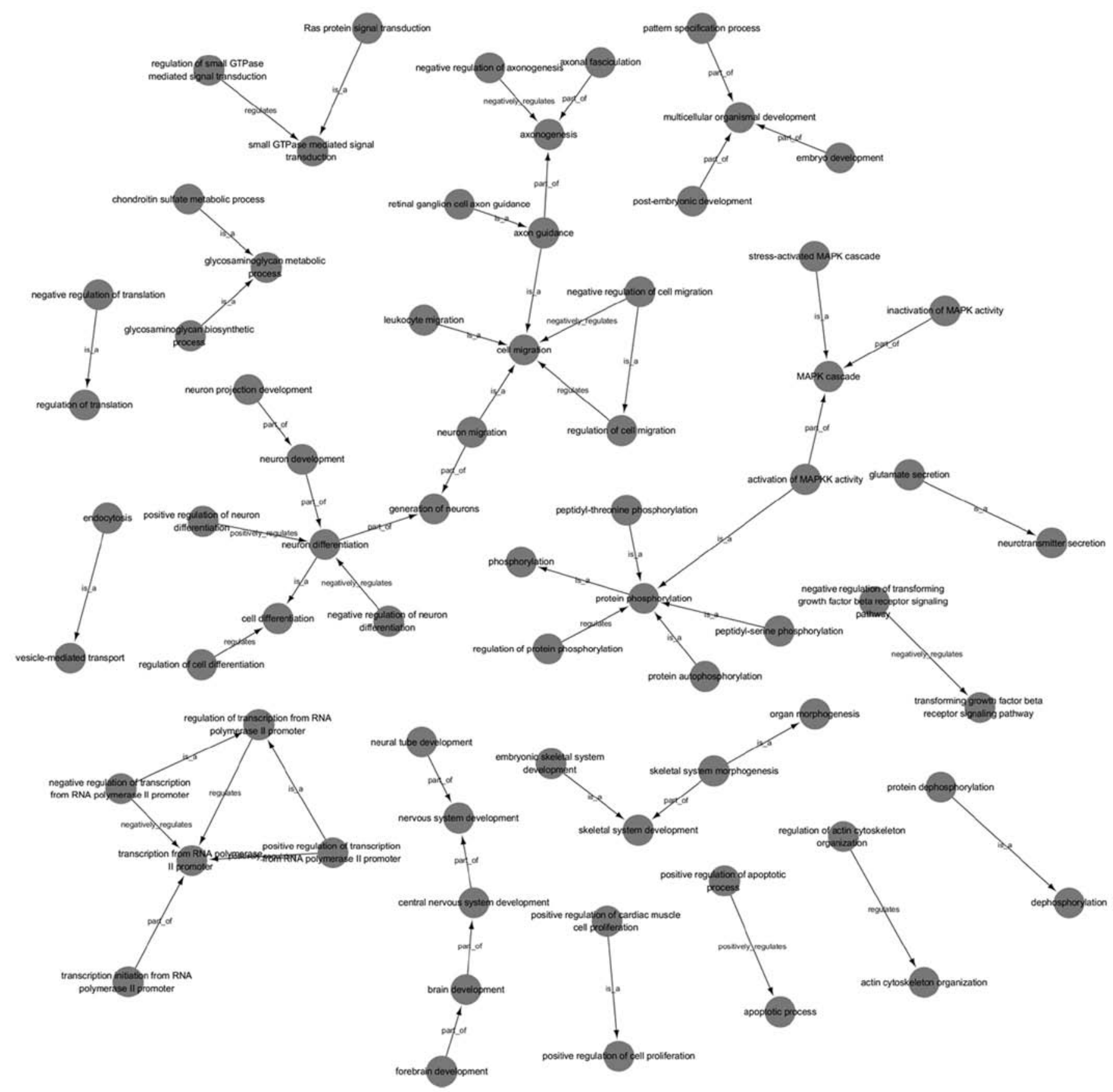

Figure 3. GO-Tree is constructed based on the Gene Ontology Directed Acyclic Graph to provide user friendly data navigation and visualization. We selected the significant GO-Term $(\mathrm{P}<0.01)$ in GO analysis based on the up and down differentially expressed genes to construct the GO-Tree to summarize the function affected in the experiment. GO, Gene Ontology.

act networks for several miRNAs target sets were frequently associated with cell signaling pathways, such as the MAPK, Wnt and PI3K-AKT signaling pathways, but also with cell migration, cytoskeleton. Thus, the significant pathway of the differential genes according to the KEGG database was idntified (Fig. 4), and the genes in the enriched biological pathway were selected $(\mathrm{P}<0.05)$. Cytoscape was used for the graphical representations of pathways (Fig. 5).

Validation of miRNA microarray results by RT-qPCR in $N S C L C$. To validate the results of miRNA microarray, miR-486-5p and miR-30a-5p were selected and assessed on the paired tissues of 40 NSCLC patients using RT-qPCR. The RT-qPCR results indicated that miR-486-5p and miR-30a-5p were significantly downregulated in NSCLCs samples used for validation, which was consistent with the results of miRNA microarray (Fig. 6).

Expression of $\mathrm{miR}-486-5 \mathrm{p}$ and $\mathrm{miR}-30 \mathrm{a}-5 \mathrm{p}$ in relation to clinicopathological characteristics. Patient characteristics with respect to the decreased expression of miR-486-5p and miR-30a-5p are shown in Table I. miR-486-5p and miR30a-5p exhibited no association with patient age, gender, histology, lymph node status, smoking history and distant 


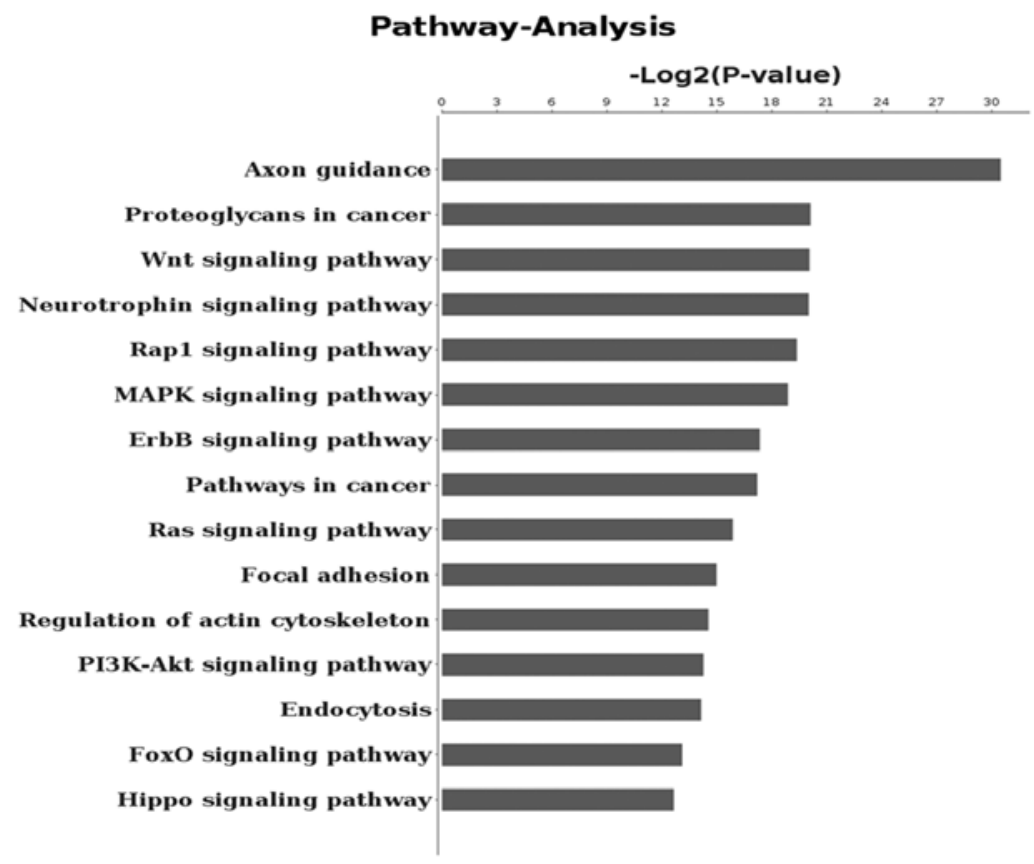

Figure 4. Pathway analysis was used to identify the significant pathway of the differential genes according to the KEGG database. We used the Fisher's exact test to select the significant pathway, and the threshold of significance was defined by p-value and FDR.

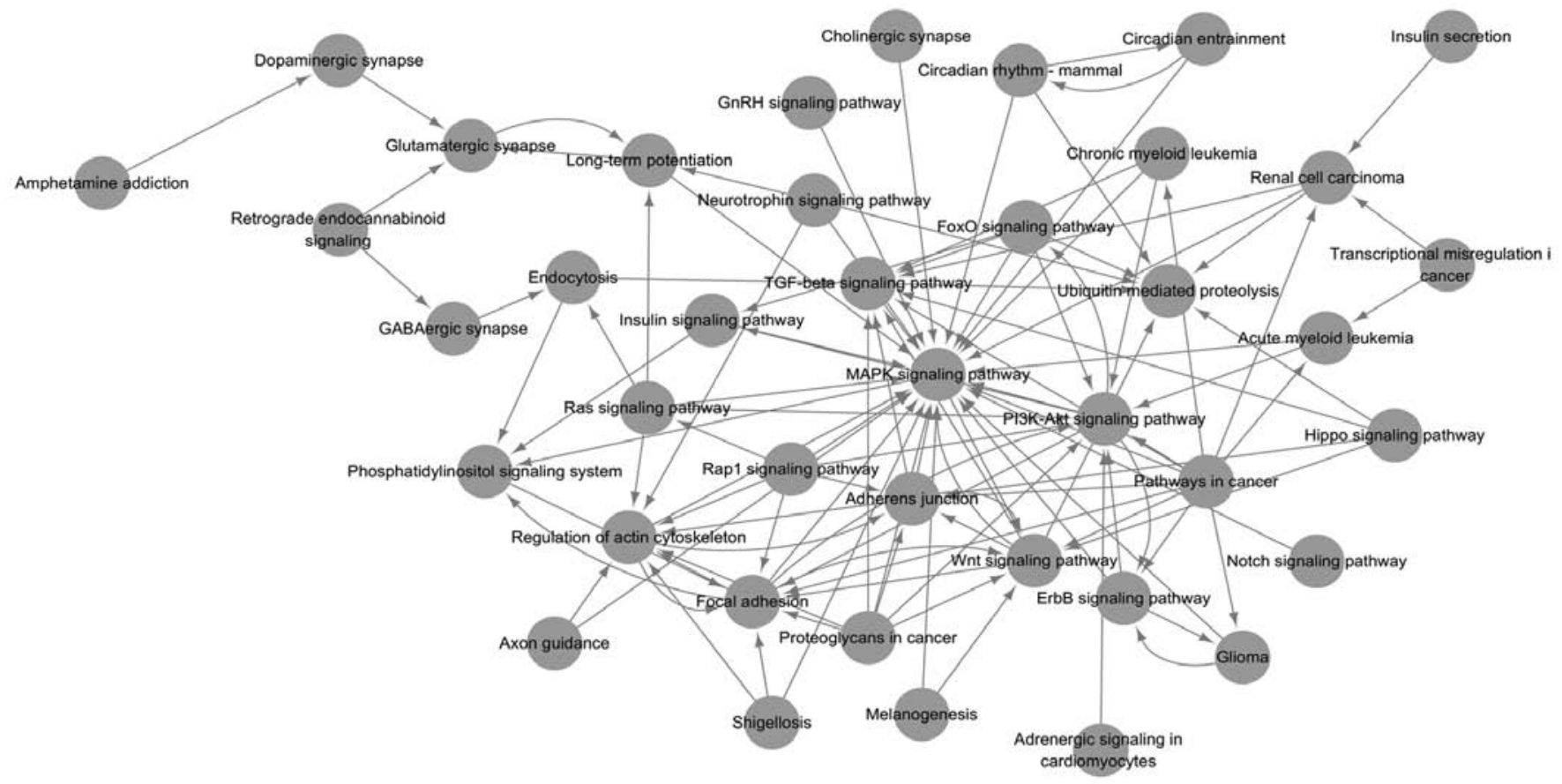

Figure 5. KEGG included metabolism, membrane transport, signal transduction and cell cycle pathways. We picked the genes in enriched biological pathway $(\mathrm{P}<0.05)$ and using Cytoscape for graphical representations of pathways.

metastases. To a certain degree, these results suggeseted that the changes in miR-486-5p or miR-30a-5p may occur at the early stage of tumorigenesis, rather than during cancer progression or metastasis.

\section{Discussion}

An incremental improvement in the survival rate of patients with cancer has been observed over the last several decades.
However, survival advances identified in other common malignancies have not been realized in lung cancer, which remains the leading cause of cancer mortality worldwide (1). The current 5-year survival rate for lung cancer is only $15 \%$.

Up to $25 \%$ of all lung cancer cases are not associated with smoking and this proportion is expected to increase as the number of smoking-associated cancer types decreases. The molecular genetic alterations including genetic and epigenetic ones occur prior to morphological changes that can be 
Table I. Demographic and clinical characteristics of NSCLC patients and the association with miR-486-5p and miR-30a-5p expression in tumor tissue specimens.

\begin{tabular}{|c|c|c|c|c|c|c|c|c|}
\hline \multirow[b]{2}{*}{ Characteristics } & \multicolumn{2}{|c|}{ All patients } & \multicolumn{3}{|c|}{$\begin{array}{l}\text { Patients with lower } \\
\text { miR-30a-5p expression }\end{array}$} & \multicolumn{3}{|c|}{$\begin{array}{l}\text { Patients with lower } \\
\text { miR-486-5p expression }\end{array}$} \\
\hline & $\mathrm{n}$ & $\%$ & $\mathrm{n}$ & $\%$ & P-value ${ }^{a}$ & $\mathrm{n}$ & $\%$ & P-value ${ }^{a}$ \\
\hline All patients & 40 & & 34 & 85.0 & & 35 & & \\
\hline \multicolumn{9}{|l|}{ Age (years) } \\
\hline$\geq 60$ & 29 & 72.5 & 25 & 73.5 & 0.921 & 26 & 74.3 & 0.862 \\
\hline$<60$ & 11 & 27.5 & 9 & 26.5 & & 9 & 25.7 & \\
\hline \multicolumn{9}{|l|}{ Gender } \\
\hline Male & 28 & 70.0 & 26 & 76.5 & 0.532 & 25 & 71.4 & 0.892 \\
\hline Female & 12 & 30.0 & 8 & 23.5 & & 10 & 28.6 & \\
\hline \multicolumn{9}{|l|}{ Histology } \\
\hline Adenocarcinomas & 20 & 50.0 & 14 & 41.2 & 0.448 & 16 & 45.7 & 0.711 \\
\hline Squamous cell carcinomas & 20 & 50.0 & 20 & 58.8 & & 19 & 54.3 & \\
\hline \multicolumn{9}{|l|}{ Smokers } \\
\hline Yes & 26 & 65.0 & 24 & 70.6 & 0.609 & 23 & 65.7 & 0.948 \\
\hline No & 14 & 35.0 & 10 & 29.4 & & 12 & 34.3 & \\
\hline \multicolumn{9}{|l|}{ Degree of differentiation } \\
\hline Low & 17 & 42.5 & 17 & 50.0 & 0.519 & 17 & 48.6 & 0.598 \\
\hline Middle & 23 & 57.5 & 17 & 50.0 & & 18 & 51.4 & \\
\hline \multicolumn{9}{|l|}{ Lymph node metastasis } \\
\hline No & 23 & 52.5 & 19 & 55.9 & 0.889 & 19 & 54.3 & 0.780 \\
\hline Yes & 17 & 42.5 & 15 & 44.1 & & 16 & 45.7 & \\
\hline \multicolumn{9}{|l|}{ Distant metastasis } \\
\hline No & 33 & 82.5 & 29 & 85.3 & 0.745 & 30 & 85.7 & 0.705 \\
\hline Yes & 7 & 17.5 & 5 & 14.7 & & 5 & 14.3 & \\
\hline \multicolumn{9}{|l|}{ Histology grade } \\
\hline $\mathrm{I} / \mathrm{II}$ & 21 & 52.5 & 16 & 47.1 & 0.641 & 17 & 48.6 & 0.734 \\
\hline III/IV & 19 & 47.5 & 18 & 52.9 & & 18 & 51.4 & \\
\hline
\end{tabular}

${ }^{\mathrm{a} C h i}$-square test; NSCLC, non-small cell lung cancer.
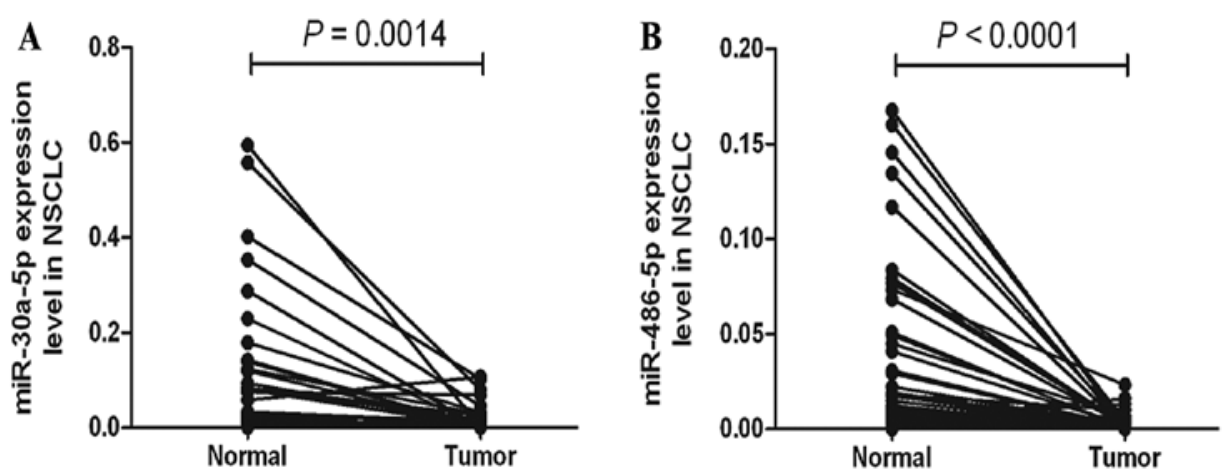

Figure 6. RT-qPCR detection of miR-30a-5p and miR-486-5p expression in lung cancer. (A) miR-30a-5p and (B) miR-486-5p were significantly downregulated in NSCLC samples used for validation,. N, adjacent non-cancerous lung tissues; $\mathrm{T}$, cancerous tissues prepared form the same patients.

identified by a cytological test (31-34). miRNA expression has been directly profiled in lung cancer, and unique groups of miRNAs were identified to characterize the neoplastic tissues or identify patients with poor prognosis $(10,35-37)$.
In this study, we used miRNA expression arrays to determine the miRNA profiles for NSCLC and paired adjacent non-cancerous lung tissues. The miRNA expression profiles distinguished NSCLC from normal lung tissue, and the 
samples were classified into two clusters: normal and NSCLC. The expression levels of miR-486-5p and miR-30a-5p were determined by RT-qPCR and consistent with the results of miRNA microarray in 80 samples.

Among miRNAs identified $(\mathrm{P}<0.005)$, a few of the miRNAs, including miR-21 and miR-200b were found to be upregulated in NSCLCs. These results were consistent with those of Tan et al and Yu et al $(17,38)$. miR-21 is one of the best-studied miRNAs, and more extensive efforts have been taken to identify the downstream genes and gene networks regulated by miR-21 and the upstream factors that can regulate dysfunction of miR-21. miR-21 can serve as an important biomarker for the early detection of lung cancer (38), and a high level of miR-21 is associated with worse prognosis of lung cancer patients. miR-200b, located on chromosome $1 \mathrm{p} 36.33$, is one of the most common regions with genomic amplications in solid tumors including lung cancer (39). Although the biological mechanism of miR-200b dysfunction in lung tumorigenesis is unclear, miR-200b has been recently identified as one of a set of miRNAs whose aberrant expression was associated with recurrence of stage I NSCLC after surgical resection (40). Consistently, we found that miR-200b overexpression existed in microRNA expression profiles data.

By contrast, a few of the miRNAs, including miR-486-5p and $\mathrm{miR}-30 \mathrm{a}-5 \mathrm{p}$ were found to be significantly downregulated in NSCLCs. miR-486 is located on one of the most frequent genomic rearrangement regions, chromosome 8p11.21, which contains potential tumor-suppressor genes in lung tumorigenesis. Recent findings have shown miR-486-5p plays a critical role in breast and lung cancer by targeted PIM-1 $(41,42)$. The results from the present study are consistent with those of previous findings, suggesting that miR-486-5p is a potential tumor suppressor in carcinogenesis. miR-30a-5p is a member of the microRNA-30 family, located on chromosome $6 \mathrm{q} 13$, which has been reported to be a genomic fragile region due to loss of heterozygosity in breast and lung cancer $(20,21)$. Zhang et al showed that miR-30a-5p can suppress breast tumor growth and metastasis by targeting metadherin (43). In lung cancer, Kumarswamy et al showed that miR-30a is downregulated in NSCLC and inhibits EMT by targeting Snail (44).

It has been widely accepted the tumorigenesis is a heterogeneous disease and develops from complex and multistep processes, including lung cancer. Evidence suggests that multiple signaling pathways involve the development of lung cancer. A meta-analysis of microRNA expression in lung cancer suggested that significant miRNAs are regulatory drivers of the oncogenic process, and may be good candidates for the development of tests for monitoring remission during postoperative follow-up, this is supported by the gene enrichment analysis (45). In our study, based on the miRNA profiles in lung cancer, an enrichment analysis was carried out, and the results showed differentially expressed miRNAs associated with the biological process and signaling pathway in lung cancer.

In conclusion, based on the miRNA array combined with the physical interaction analysis, our results have shown the impact of significant miRNAs on cell pathways and biological processes. Further validation of miRNA microarray results by RT-qPCR in NSCLC show that miR-486-5p and miR$30 \mathrm{a}-5 \mathrm{p}$ were significantly downregulated in NSCLC tissues. Therefore, the results provide strong evidence of the possible involvement of miRNAs in the development and progression of NSCLC, The findings are of importance for both clinical investigators and for those who design miRNA-based novel cancer therapeutics.

\section{Acknowledgements}

We are grateful for the participation and cooperation from the patients with NSCLC. This study was supported by grants from the National Natural Science Foundation of China (31270940 to J.-A. Huang; 81201575 to Z.-Y. Liu), the Science and Technology Committee of Jiangsu Province (BK2012606 to J.-A. Huang), the Jiangsu Province Colleges and Universities Natural Science Research Foundation (12KJB310016 to Z.-Y. Liu), and the Foundation of Health Care Rejuvenation by Science and Education (KJXW2011006 to Z.-Y. Liu) and Clinical Key Speciality Project of China.

\section{References}

1. Jemal A, Bray F, Center MM, Ferlay J, Ward E and Forman D: Global cancer statistics. CA Cancer J Clin 61: 69-90, 2011.

2. Wang T, Nelson RA, Bogardus A and Grannis FW Jr: Five-year lung cancer survival: Which advanced stage nonsmall cell lung cancer patients attain long-term survival? Cancer 116: 1518-1525, 2010.

3. Guo H, Ingolia NT, Weissman JS and Bartel DP: Mammalian microRNAs predominantly act to decrease target mRNA levels. Nature 466: 835-840, 2010.

4. Wilson RC and Doudna JA: Molecular mechanisms of RNA interference. Annu Rev Biophys 42: 217-239, 2013.

5. Lewis BP, Burge CB and Bartel DP: Conserved seed pairing, often flanked by adenosines, indicates that thousands of human genes are microRNA targets. Cell 120: 15-20, 2005.

6. Bueno MJ, Pérez de Castro I and Malumbres M: Control of cell proliferation pathways by microRNAs. Cell Cycle 7: 3143-3148, 2008.

7. Lee CT, Risom T and Strauss WM: MicroRNAs in mammalian development. Birth Defects Res C Embryo Today 78: 129-139, 2006.

8. Jovanovic M and Hengartner MO: miRNAs and apoptosis: RNAs to die for. Oncogene 25: 6176-6187, 2006.

9. Lu J, Getz G, Miska EA, Alvarez-Saavedra E, Lamb J, Peck D, Sweet-Cordero A, Ebert BL, Mak RH, Ferrando AA, et al: MicroRNA expression profiles classify human cancers. Nature 435: 834-838, 2005.

10. Lebanony D, Benjamin H, Gilad S, Ezagouri M, Dov A, Ashkenazi K, Gefen N, Izraeli S, Rechavi G, Pass H, et al: Diagnostic assay based on hsa-miR-205 expression distinguishes squamous from nonsquamous non-small-cell lung carcinoma. J Clin Oncol 27: 2030-2037, 2009.

11. Markou A, Tsaroucha EG, Kaklamanis L, Fotinou M, Georgoulias V and Lianidou ES: Prognostic value of mature microRNA-21 and microRNA-205 overexpression in non-small cell lung cancer by quantitative real-time RT-PCR. Clin Chem 54: 1696-1704, 2008

12. Gregory PA, Bert AG, Paterson EL, Barry SC, Tsykin A, Farshid G, Vadas MA, Khew-Goodall Y and Goodall GJ: The miR-200 family and miR-205 regulate epithelial to mesenchymal transition by targeting ZEB1 and SIP1. Nat Cell Biol 10: 593-601, 2008.

13. Chao $\mathrm{CH}$, Chang $\mathrm{CC}$, Wu MJ, Ko HW, Wang D, Hung MC, Yang JY and Chang CJ: MicroRNA-205 signaling regulates mammary stem cell fate and tumorigenesis. J Clin Invest 124: 3093-3106, 2014.

14. Midorikawa Y, Yamamoto S, Tsuji S, Kamimura N, Ishikawa S, Igarashi H, Makuuchi M, Kokudo N, Sugimura H and Aburatani H: Allelic imbalances and homozygous deletion on 8p23.2 for stepwise progression of hepatocarcinogenesis. Hepatology 49: 513-522, 2009.

15. Jiang F, Yin Z, Caraway NP, Li R and Katz RL: Genomic profiles in stage I primary non small cell lung cancer using comparative genomic hybridization analysis of cDNA microarrays. Neoplasia 6: 623-635, 2004. 
16. Shen J, Liu Z, Todd NW, Zhang H, Liao J, Yu L, Guarnera MA, Li R, Cai L, Zhan M, et al: Diagnosis of lung cancer in individuals with solitary pulmonary nodules by plasma microRNA biomarkers. BMC Cancer 11: 374, 2011.

17. Tan X, Qin W, Zhang L, Hang J, Li B, Zhang C, Wan J, Zhou F, Shao K, Sun Y, et al: A 5-microRNA signature for lung squamous cell carcinoma diagnosis and hsa-miR-31 for prognosis. Clin Cancer Res 17: 6802-6811, 2011.

18. Ragusa M, Majorana A, Statello L, Maugeri M, Salito L, Barbagallo D, Guglielmino MR, Duro LR, Angelica R, Caltabiano R, et al: Specific alterations of microRNA transcriptome and global network structure in colorectal carcinoma after cetuximab treatment. Mol Cancer Ther 9: 3396-3409, 2010.

19. Wang J, Tian X, Han R, Zhang X, Wang X, Shen H, Xue L, Liu Y, Yan X, Shen J, et al: Downregulation of miR-486-5p contributes to tumor progression and metastasis by targeting protumorigenic ARHGAP5 in lung cancer. Oncogene 33: 1181-1189, 2014.

20. Noviello C, Courjal $\mathrm{F}$ and Theillet C: Loss of heterozygosity on the long arm of chromosome 6 in breast cancer: Possibly four regions of deletion. Clin Cancer Res 2: 1601-1606, 1996.

21. Hu XY, Bai XM, Qiao X and Zhu YQ: Copy number variation at $6 \mathrm{q} 13$ is associated with lung cancer risk in a Han Chinese population. Exp Lung Res 39: 427-433, 2013.

22. Agrawal R, Tran U and Wessely O: The miR-30 miRNA family regulates Xenopus pronephros development and targets the transcription factor Xlim1/Lhx1. Development 136: 3927-3936, 2009.

23. Zhu H, Wu H, Liu X, Li B, Chen Y, Ren X, Liu CG and Yang JM: Regulation of autophagy by a beclin 1-targeted microRNA, miR-30a, in cancer cells. Autophagy 5: 816-823, 2009.

24. Wettenhall JM and Smyth GK: limmaGUI: A graphical user interface for linear modeling of microarray data. Bioinformatics 20: 3705-3706, 2004.

25. Benjamini Y, Drai D, Elmer G, Kafkafi N and Golani I: Controlling the false discovery rate in behavior genetics research. Behav Brain Res 125: 279-284, 2001.

26. Ashburner M, Ball CA, Blake JA, Botstein D, Butler H, Cherry JM, Davis AP, Dolinski K, Dwight SS, Eppig JT, et al; The Gene Ontology Consortium: Gene ontology: Tool for the unification of biology. Nat Genet 25: 25-29, 2000 .

27. Zhang B, Schmoyer D, Kirov S and Snoddy J: GOTree Machine (GOTM): A web-based platform for interpreting sets of interesting genes using Gene Ontology hierarchies. BMC Bioinformatics 5: 16, 2004.

28. Draghici S, Khatri P, Tarca AL, Amin K, Done A, Voichita C, Georgescu C and Romero R: A systems biology approach for pathway level analysis. Genome Res 17: 1537-1545, 2007.

29. Shannon P, Markiel A, Ozier O, Baliga NS, Wang JT, Ramage D, Amin N, Schwikowski B and Ideker T: Cytoscape: A software environment for integrated models of biomolecular interaction networks. Genome Res 13: 2498-2504, 2003.

30. Varkonyi-Gasic E, Wu R, Wood M, Walton EF and Hellens RP: Protocol: A highly sensitive RT-PCR method for detection and quantification of microRNAs. Plant Methods 3: 12, 2007.

31. Belinsky SA, Liechty KC, Gentry FD, Wolf HJ, Rogers J, Vu K, Haney J, Kennedy TC, Hirsch FR, Miller Y, et al: Promoter hypermethylation of multiple genes in sputum precedes lung cancer incidence in a high-risk cohort. Cancer Res 66: 3338-3344, 2006.
32. Jiang F, Todd NW, Qiu Q, Liu Z, Katz RL and Stass SA: Combined genetic analysis of sputum and computed tomography for noninvasive diagnosis of non-small-cell lung cancer. Lung Cancer 66: 58-63, 2009

33. Liu Z, Zhao J, Chen XF, Li W, Liu R, Lei Z, Liu X, Peng X, Xu K, Chen $\mathrm{J}$, et al: $\mathrm{CpG}$ island methylator phenotype involving tumor suppressor genes located on chromosome $3 \mathrm{p}$ in non-small cell lung cancer. Lung Cancer 62: 15-22, 2008.

34. Liu Z, Li W, Lei Z, Zhao J, Chen XF, Liu R, Peng X, Wu ZH, Chen J, Liu H, et al: $\mathrm{CpG}$ island methylator phenotype involving chromosome $3 p$ confers an increased risk of non-small cell lung cancer. J Thorac Oncol 5: 790-797, 2010.

35. Yu SL, Chen HY, Chang GC, Chen CY, Chen HW, Singh S, Cheng CL, Yu CJ, Lee YC, Chen HS, et al: MicroRNA signature predicts survival and relapse in lung cancer. Cancer Cell 13: 48-57, 2008.

36. Yanaihara N, Caplen N, Bowman E, Seike M, Kumamoto K, Yi M, Stephens RM, Okamoto A, Yokota J, Tanaka T, et al: Unique microRNA molecular profiles in lung cancer diagnosis and prognosis. Cancer Cell 9: 189-198, 2006.

37. Takamizawa J, Konishi H, Yanagisawa K, Tomida S, Osada $H$, Endoh H, Harano T, Yatabe Y, Nagino M, Nimura Y, et al: Reduced expression of the let-7 microRNAs in human lung cancers in association with shortened postoperative survival. Cancer Res 64: 3753-3756, 2004.

38. Yu L, Todd NW, Xing L, Xie Y, Zhang H, Liu Z, Fang H, Zhang J, Katz RL and Jiang F: Early detection of lung adenocarcinoma in sputum by a panel of microRNA markers. Int J Cancer 127: 2870-2878, 2010

39. Scaruffi P, Parodi S, Mazzocco K, Defferrari R, Fontana V, Bonassi S and Tonini GP: Detection of MYCN amplification and chromosome 1p36 loss in neuroblastoma by cDNA microarray comparative genomic hybridization. Mol Diagn 8: 93-100, 2004.

40. Patnaik SK, Kannisto E, Knudsen S and Yendamuri S: Evaluation of microRNA expression profiles that may predict recurrence of localized stage I non-small cell lung cancer after surgical resection. Cancer Res 70: 36-45, 2010.

41. Zhang G, Liu Z, Cui G, Wang X and Yang Z: MicroRNA-486-5p targeting PIM-1 suppresses cell proliferation in breast cancer cells. Tumour Biol 35: 11137-11145, 2014.

42. Pang W, Tian X, Bai F, Han R, Wang J, Shen H, Zhang X, Liu Y, Yan X, Jiang F, et al: Pim-1 kinase is a target of miR-486-5p and eukaryotic translation initiation factor $4 \mathrm{E}$, and plays a critical role in lung cancer. Mol Cancer 13: 240, 2014.

43. Zhang N, Wang X, Huo Q, Sun M, Cai C, Liu Z, Hu G and Yang Q: MicroRNA-30a suppresses breast tumor growth and metastasis by targeting metadherin. Oncogene 33: 3119-3128, 2014.

44. Kumarswamy R, Mudduluru G,Ceppi P,Muppala S, Kozlowski M, Niklinski J, Papotti M and Allgayer H: MicroRNA-30a inhibits epithelial-to-mesenchymal transition by targeting Snail and is downregulated in non-small cell lung cancer. Int J Cancer 130: 2044-2053, 2012.

45. Võsa U, Vooder T, Kolde R, Vilo J, Metspalu A and Annilo T: Meta-analysis of microRNA expression in lung cancer. Int $\mathrm{J}$ Cancer 132: 2884-2893, 2013. 